\title{
Comparison of Medroxyprogesterone Acetate With Cetrotide for Prevention of Premature Luteinizing Hormone Surges in Women Undergoing In Vitro Fertilization
}

\author{
Kobra Hamdi ${ }^{1}$, Laya Farzadi ${ }^{1}$, Alea Ghasemzadeh ${ }^{1}$, Nazli Navali ${ }^{1}$, Simin Atashkhoei ${ }^{1}$, Helen Pia ${ }^{1}$, \\ Vahedeh Shahnazi ${ }^{1}$, Amir Fattahi ${ }^{2}$, Zahra Bahrami-asl ${ }^{1}$, Farnaz Sepasi ${ }^{1}$, Massomeh Dopour Faliz ${ }^{1}$, \\ Nahid Lahroudi ${ }^{1}$, Parvin Hakimi ${ }^{{ }^{*}}$
}

\begin{abstract}
Objectives: The aim of the study was to investigate the role of medroxyprogesterone acetate (MPA) in the prevention of luteinizing hormone (LH) surge during controlled ovarian hyperstimulation $(\mathrm{COH})$. Characteristics of cycle and pregnancy outcomes were compared in subsequent frozen-thawed embryo transfer (FET) cycles.

Materials and Methods: In a prospective controlled study, In vitro fertilization (IVF)/intracytoplasmic sperm injection treatment was done in 99 patients. In the case group, hMG and MPA were administered from third day of the cycle, simultaneously. As dominant follicles matured, ovulation was induced by hCG or GnRH agonist. hMG and GnRH were administrated to the control group. For later transfer in both protocols, viable embryos were cryopreserved. The primary outcome measured was the incidence of premature LH surge and a number of oocytes retrieved. Clinical pregnancy outcomes from FETs were secondary outcomes.

Results: The number of oocytes retrieved in both case and control groups were equal. LH suppression persisted during ovarian stimulation in the case group, and there was no incidence of premature LH surge. There was no significant difference between amounts of follicles, mature follicles, oocytes resumed and obtained embryos between 2 groups $(P>0.05)$.

Conclusions: The results showed that in a woman undergoing $\mathrm{COH}, \mathrm{MPA}$ as an oral drug was effective in the prevention of premature LH surge. The results would help to establish a new method for ovarian stimulation in combination with embryo cryopreservation.

Keywords: Medroxyprogesterone acetate, Controlled ovarian stimulation, Premature LH surge
\end{abstract}

\section{Introduction}

The prevalence of infertility is $16.7 \%$ worldwide, and characteristics and causes of infertility are different between industrialized, developed and developing countries $(1,2)$. Diagnosis of infertility results in choosing appropriate therapeutic approach in terms of time, and cost is the necessity of treatment planning $(1,2)$.

In vitro fertilization (IVF) is a fully coordinated sequence of procedures that begins with severe and controlled stimulation of ovaries using exogenous gonadotropin and is followed by resumption of oocytes from ovaries under the guide of transvaginal sonography; thereafter the process is continued by IVF and transfer of the embryo in a trans-cervical manner into the uterus $(3,4)$.

Surge or sudden early peak of luteinizing hormone (LH) is one of the causes of cycle cancelation during controlled ovarian stimulation in patients undergoing IVF. LH secretion stimulates ovulation during the normal cycle in response to a rapid increase of estradiol concentration. However, an early surge of LH can put the oocyte resumption at risk during IVF cycle (5). Based on the previous findings, incidence of the early LH surge among patients is totally different and varies between $0.34 \%$ and $38 \%$, although there is growing evidence that elder patients with reduced ovarian reserve are at great risk of early LH surge (6).

Increased concentration of LH in early stages of follicle development could have adverse influences, especially on the pregnancy rate. Many efforts have been done to reduce the incidence of early $\mathrm{LH}$ surge including various stimulatory protocols by $\mathrm{GnRH}$ agonists and antagonists. In this term, it has been documented that desensitization by $\mathrm{GnRH}$ antagonists could increase follicular synchronization $(5,7,8)$. Antagonist protocol also could cause rapid and reversible LH suppression without early flare effect (6-9). On the other hand, it has been suggested that adjuvant therapy with GnRH agonists could overcome early luteinization and need for repeated measurements of serum LH. Although, adjuvant therapy with GnRH agonist could sometimes reduce response to 
stimulation and so high dose of the drug as well as long treatment duration would be required to reach appropriate follicular development (9). In such cases, in addition to higher medication costs, there is a risk of ovarian hyperstimulation syndrome (OHSS), since higher frequency of OHSS has been observed in patients under long-term agonist compared to antagonist (10). Prolonged treatment cycle and repeated daily injections of GnRH agonists is the main fault of this method which has caused more use of GnRH antagonist protocol in most of the infertility medical centers $(8,11,12)$.

Previous studies have shown that medroxyprogesterone acetate (MPA) could be an alternative treatment in preventing LH early surge in patients undergoing IVF treatment. Other advantages of this drug, in addition to beneficial effect on serum LH, include low cost, easy use (oral consumption), and availability $(6,10)$.

Nowadays, Cetrotide ${ }^{\circledR}$ (Cetrorelix acetate), a kind of GnRH antagonist, is used as an LH surge inhibitor in patients under IVF protocol and has a risk of OHSS. Although it should be mentioned that in Iran, prevalence of using injectable form of the drug as well as its high cost and availability problems could be considered as the drug disadvantages.

Now oral form of MPA is available which is not only costeffective than Cetrotide, but also has a better acceptance among the patients and several studies have shown its beneficial effect on the prevention of moderate and severe OHSS (13). However, necessity of embryo freezing and embryo transferring in next cycles is still an issue for this medication. Based on previous findings, transferring the embryo in next cycles could increase implantation and pregnancy rates (14). Furthermore, in the case of oocyte donation in which the endometrial condition of the donor woman is not considered, MPA could be an appropriate medication with the ability of preventing early LH surge.

\section{Materials and Methods}

Patients were screened by transvaginal sonography and their FSH and estradiol levels were evaluated during the third day of menstruation in serum, then they were divided to 2 case and control groups. Inclusion criteria were: infertile women aged 20-40 years, AFC 4 or at least 4 on the third day of menstrual cycle, and FSH lower than $15 \mathrm{IU} / \mathrm{L}$.

Exclusion criteria included evidence of ovarian failure (FSH rate above 15 IU/L or lack of AFC in sonography evaluation on the third day of menstruation), grade 3 or 4 endometriosis, every contraindication for ovarian stimulation, and severe male factor.

Recombinant FSH (rFSH- Gonal-f Merck Serono) 150$225 \mathrm{IU}$ and MPA $10 \mathrm{mg} / \mathrm{d}$ were prescribed to the case group, from the third day of menstruation cycle. Follicular monitoring began on the 5th-6th day of stimulation by sonography and repeated every 2-3 days according to the patients' situations.
Serum levels of progesterone, estradiol, FSH, and $\mathrm{LH}$ were evaluated in the blood samples during third menstruation day (Table 1). In addition, LH, progesterone and estradiol were measured on the day of hCG (human chorionic gonadotropin) injection (Table 2). Progesterone and LH were measured along with sonography. Since 3 follicles were seen above $17 \mathrm{~mm}, 10000 \mathrm{IU}$ of hCG or $0.2 \mathrm{mg}$ Decapeptyl was prescribed for final maturation of oocytes and ovulation. In the case of probably OHSS, oocyte resumption was performed under transvaginal sonography guide and general anesthesia after 34-36 hours after stimulation of ovulation by hCG and the follicles the size above 13-14 $\mathrm{mm}$ in diameter were retrieved. In the control group receiving stimulatory protocol with antagonists, stimulation by gonadotropin was performed since the third day of menstruation. Treatment by $\mathrm{rFSH}$ (Gonal-f Merck Serono) 150-225 IU/d was performed from the third day of the cycle. Transvaginal sonography was performed 5-6 days after stimulation by gonadotropin and then repeated according to the patient's situation every 2-3 days. GnRH antagonist like Cetrotide 0.25 $\mathrm{mg} / \mathrm{d}$ (Merck Serono) was subcutaneously administered when follicular diameter reached above 13-14 mm. By appearance of 3 follicles larger than $17 \mathrm{~mm}$ in diameter, final maturation of oocytes and trigger of ovulation were done using 10000 IU hCG or 0.2 mg Decapeptyl. Aspirated oocytes were fertilized in vitro by ICSI (intracytoplasmic sperm injection).

Embryos were evaluated according to Cummins criteria on the third day of fertilization by number, and regularity of blastomeres and embryonic fragmentation grade. All qualified embryos (8 cell of grade 1 and 2 embryos) were frozen using viterification mechanism on the third day of oocyst retrieved. The vitrification mechanism was utilized to freeze oocytes using Cryotop carrier system. For defreezing the embryos sucrose solution at a concentration of $0,0.5$ and $1 \mathrm{M}$ were used intermittently as a diluent for protectant solution.

The number of retrieved oocytes, viable embryos, their grade, and pregnancy test results were compared between two groups. Endometrial preparation was performed in FET cycle using the same method for two groups. On the second day of cycle, administration of $6 \mathrm{mg}$ estradiol continued, until the endometrial thickness reached above $7 \mathrm{~mm}$. Then progesterone administration was started $(100 \mathrm{mg} / \mathrm{IM})$ and on the fourth day after progesterone administration, embryos were transferred and estradiol and progesterone $(300 \mathrm{mg} / \mathrm{d})$ were prescribed to support

Table 1. Hormonal Changes in Both Groups During Day 3 (Mean \pm SE)

\begin{tabular}{lcccc}
\hline \multirow{2}{*}{ Group } & \multicolumn{4}{c}{ Day 3 } \\
\cline { 2 - 5 } & FSH & Estradiol & LH & Progesterone \\
\hline Case & $6.19 \pm 0.49$ & $62.79 \pm 5.09$ & $5.04 \pm 0.41$ & $0.88 \pm 0.23$ \\
Control & $5.28 \pm 0.28$ & $73.79 \pm 8.40$ & $4.47 \pm 0.37$ & $0.66 \pm 0.15$ \\
$P$ value & 0.113 & 0.269 & 0.303 & 0.440 \\
\hline
\end{tabular}


Table 2. Hormonal Changes in Both Groups During Follicular Maturation and hCG Injection (Mean \pm SE)

\begin{tabular}{|c|c|c|c|c|c|}
\hline \multirow{2}{*}{ Group } & \multicolumn{2}{|c|}{ During Follicular Maturation } & \multicolumn{3}{|c|}{ During hCG Injection } \\
\hline & Progesterone & LH & Progesterone & LH & Estradiol \\
\hline Case & $1.02 \pm 0.05$ & $4.81 \pm 0.37$ & $1.27 \pm 0.23$ & $4.12 \pm 0.67$ & $1970.30 \pm 156.49$ \\
\hline Control & $0.95 \pm 0.12$ & $4.12 \pm 0.21$ & $1.01 \pm 0.22$ & $3.06 \pm 0.51$ & $2259.90 \pm 172.60$ \\
\hline$P$ value & 0.602 & 0.401 & 0.303 & 0.216 & 0.218 \\
\hline
\end{tabular}

luteal phase continuously.

If implantation was successful, prescription of estradiol and progesterone would continue until 12 weeks of pregnancy otherwise it was terminated. If there was no growth in follicles, the cycle was canceled.

The results were analyzed by SPSS statistical software version 16.0. Independent $t$ test was used for evaluation of the results. Qualitative measures were demonstrated in the form of frequency and percentage and quantitative measures were described as mean \pm standard error.

\section{Results}

The control and case groups included 50 and 49 patients, respectively. The average age of control group was $31 \pm 6$ and case group was $30 \pm 6$, and there were no significant differences between them $(P=0.28)$.

In the case group, of 49 patients, 6 were excluded from the study because of insufficient response. Although, one patient was excluded from the case group because of drug refusal and 2 patients as there were no available embryos for transfer. In the control group, 7 patients were excluded from the study because of insufficient response to the treatment and undeveloped follicles and 2 patients were excluded as there were no available embryos for transfer.

Infertility duration in the control and case groups were $7.04 \pm 0.78$ and $8.06 \pm 0.75$ years, respectively, and there were no significant differences $(P=0.35)$ between them. Follicles above $17 \mathrm{~mm}$ were $14.40 \pm 0.87$ in the control group and $13.69 \pm 0.84$ in the case group $(P=0.55)$. Mature follicles (above $17 \mathrm{~mm}$ ) were $12.09 \pm 0.88$ and $11.47 \pm 0.79$ in the control and case groups, respectively $(P=0.60)$. Retrieved oocytes were $9.95 \pm 0.91$ and $10.02 \pm 0.88$ in the control and case groups, respectively $(P=0.95)$. The number of the embryos were $6.85 \pm 0.56$ and $6.92 \pm 0.63$ in the control and case groups, respectively $(P=0.93)$. The endometrial diameter was $7.71 \pm 0.23 \mathrm{~mm}$ in the control and $8.20 \pm 0.12 \mathrm{~mm}$ in the case group without significant differences $(P=0.07)$. The rate of successful pregnancies was $27 \%$ in the case and $23 \%$ in the control groups $(P=0.21)$.

Primary infertility was significantly different between the groups and included $74 \%$ of the control group and $91 \%$ of the case group $(P=0.01)$. Results indicated that cycle cancellation was occurred in $19 \%$ of patients in whom average duration of infertility was estimated $8.05 \pm 4.61$ years and in successful patients, the duration was $7.42 \pm 5.63$ years. These results showed there was no relationship between infertility duration and cycle cancellation in the cases $(P=0.61)$. Premature luteinizing hormone surges was not observed in our study. In women above 30 years old, the cycle cancellation rate was higher compared to younger women $(P=0.035)$.

\section{Discussion}

Our findings demonstrated that the case group had better results in IVF cases. Results indicated that MPA could be prescribed as an alternative oral and easy access drug instead of GnRH antagonist in the patients that underwent controlled ovarian stimulation in the case of IVF. In the patients undergoing controlled ovarian stimulation for IVF, medroxyprogesterone could be used successfully as a treatment protocol. It was not used commonly earlier, but results demonstrated that in the patients to whom embryo would be transferred in the same cycle, administration of $\mathrm{GnRH}$ antagonist despite the higher costs and injectable form had no advantages in comparison to medroxyprogesterone. Researchers indicated that the use of MPA was effective in the prevention of premature LH surge in a woman who went under controlled ovarian hyperstimulation $(\mathrm{COH})(6)$.

The results of the study indicated there was no significant difference between amounts of follicles, mature follicles, oocyte number and viable embryos in 2 groups. In addition, medroxyprogesterone was a trustworthy drug and had no negative effect on the pregnancy rate, growth, development of oocytes and embryos. These results indicated that medroxyprogesterone was effective on $\mathrm{COH}$ without any complications. These results were in agreement with previous studies $(6,10)$.

Some studies reported that the increase of progesterone in the blood can interfere with the development of follicles and oocytes and reduce fertility potential. The results demonstrated that MPA at the used dose in our study had no adverse effect on the development of follicles and oocytes. It is likely to mention that our knowledge about effects of progesterone on development of oocytes and follicles was incomplete and unclear and could have adverse effects on the development of oocytes and follicles at a higher dose of medroxyprogesterone in the patients. This requires further exploration.

The effects of progestin in premature LH suppression and ovulation was unknown, although it blocked $\mathrm{LH}$ surge (induced by estradiol) through blocking the GnRH surge induction system. In our study, premature LH surge was not seen in any patient in both groups, and it seems both drugs had appropriate effects on premature 
LH surge control. The results were in agreement with Wang et al results, as they also reported there was not any premature LH surge $(6,10)$. During the normal follicular phase, progestin administration reduced plasma levels of LH in comparison to untreated groups (4). Progesterone prevented premature LH surge by affecting estradiol and GnRH cycle. Progesterone prevented activation of estradiol system induced $\mathrm{GnRH}$ surge and blocked the LH surge induced by estradiol (6). Researchers indicated that progesterone prevented induced LH surge against E2 in early stages of signal transmission $(15,16)$. Researchers reported that MPA could block premature LH surge on day 3, but the administration of MPA later during midfollicular phase was not effective (6). Moreover, MPA was used in the patients with estradiol levels $50-70 \mathrm{pg} / \mathrm{mL}$ on day 3 of the cycle (6).

Our results indicated that the infertility duration was not in relation with cycle canceling, and there was no significant difference between the groups in cycle cancellation, and infertility duration was equal in both groups. Some previous studies indicated that in the patients undergoing IVF, the infertility duration had a direct relationship with cycle cancellation rates. Although, this relation was influenced by some factors, such as the age of selected person with longer duration of infertility and this may be a risk factor for a successful cycle. It is important to be aware of such demographic characteristics as patient's body mass index (BMI), because high BMI is a risk factor for cycle cancellation. Unfortunately, this information was not available for our study, so it was not possible to judge about the relation between infertility duration and cycle cancellation rate.

According to the results, to prevent premature LH surge in IVF cases, medroxyprogesterone could be used as an appropriate medication instead of $\mathrm{GnRH}$ antagonists, because it had no complication in comparison to $\mathrm{GnRH}$ antagonists and had the same effects. Results demonstrated medroxyprogesterone would decrease premature LH surge and had no adverse effects on pregnancy outcomes in IVF patients.

One of the disadvantages in progesterone treatment is the obligation of embryo freezing for transfer to the uterus, but sometimes due to occupational or familial reasons, patients request the embryos to be frozen for transfer in the next cycle. Moreover, in the patients with inappropriate conditions of the endometrium and oocyst donation or because of hyper-stimulation in embryo transfer to the next cycle, progesterone had advantages in comparison to other methods.

\section{Conflict of Interests}

Authors declare that they have no conflict of interests.

\section{Ethical Issues}

This study was approved by the Ethics Committee of
Tabriz University of Medical Sciences (IR.TBZMED. REC.1395.1127). The protocol of the study was registered in the Iranian Registry of Clinical Trials (IRCT2017010813566N8).

\section{Financial Support}

Financial support of this study was provided by Women's Reproductive Health Research Center, Tabriz University of Medical Sciences.

\section{References}

1. Gardner DK, Weissman A, Howles CM, Shoham Z. Textbook of assisted reproductive technologies: laboratory and clinical perspectives. Italy, Dunitz Com: CRC press; 2008.

2. Lunenfeld B, Insler V. Infertility: Male and Female. Edinburgh: Churchill Livingstone; 1986.

3. Messinis IE. Ovarian feedback, mechanism of action and possible clinical implications. Hum Reprod Update. 2006;12(5):557-571. doi:10.1093/humupd/dml020

4. Soules MR, Steiner RA, Clifton DK, Cohen NL, Aksel S, Bremner WJ. Progesterone modulation of pulsatile luteinizing hormone secretion in normal women. J Clin Endocrinol Metab. 1984;58(2):378-383. doi:10.1210/jcem58-2-378

5. Wikstrom A, Green B, Johansson ED. The plasma concentration of medroxyprogesterone acetate and ovarian function during treatment with medroxyprogesterone acetate in 5 and $10 \mathrm{mg}$ doses. Acta Obstet Gynecol Scand. 1984;63(2):163-168.

6. Kuang Y, Chen Q, Fu Y, et al. Medroxyprogesterone acetate is an effective oral alternative for preventing premature luteinizing hormone surges in women undergoing controlled ovarian hyperstimulation for in vitro fertilization. Fertil Steril. 2015;104(1):62-70.e63. doi:10.1016/j.fertnstert.2015.03.022

7. Pohl CR, Richardson DW, Marshall G, Knobil E. Mode of action of progesterone in the blockade of gonadotropin surges in the rhesus monkey. Endocrinology. 1982;110(4):1454-1455. doi:10.1210/endo-110-4-1454

8. Griesinger G, Venetis CA, Marx T, Diedrich K, Tarlatzis BC, Kolibianakis EM. Oral contraceptive pill pretreatment in ovarian stimulation with $\mathrm{GnRH}$ antagonists for IVF: a systematic review and metaanalysis. Fertil Steril. 2008;90(4):1055-1063. doi:10.1016/j. fertnstert.2007.07.1354

9. Venetis CA, Kolibianakis EM, Papanikolaou E, Bontis J, Devroey P, Tarlatzis BC. Is progesterone elevation on the day of human chorionic gonadotrophin administration associated with the probability of pregnancy in in vitro fertilization? A systematic review and meta-analysis. Hum Reprod Update. 2007;13(4):343-355. doi:10.1093/humupd/ dmm007

10. Wang Y,Chen Q, WangN, Chen H, Lyu Q, KuangY.Controlled Ovarian Stimulation Using Medroxyprogesterone Acetate and hMG in Patients With Polycystic Ovary Syndrome Treated for IVF: A Double-Blind Randomized Crossover Clinical Trial. Medicine (Baltimore). 2016;95(9):e2939. doi:10.1097/md.0000000000002939 
11. Schwallie PC. The effect of depot-medroxyprogesterone acetate on the fetus and nursing infant: a review. Contraception. 1981;23(4):375-386.

12. Salehnia M, Zavareh S. The effects of progesterone on oocyte maturation and embryo development. Int J Fertil Steril. 2013;7(2):74-81.

13. Escudero EL, Boerrigter PJ, Bennink HJ, et al. Mifepristone is an effective oral alternative for the prevention of premature luteinizing hormone surges and/or premature luteinization in women undergoing controlled ovarian hyperstimulation for in vitro fertilization. J Clin Endocrinol Metab. 2005;90(4):2081-2088. doi:10.1210/jc.2004-1160
14. Yu Ng EH, Yeung WS, Yee Lan Lau E, So WW, Ho PC. High serum oestradiol concentrations in fresh IVF cycles do not impair implantation and pregnancy rates in subsequent frozen-thawed embryo transfer cycles. Hum Reprod. 2000;15(2):250-255.

15. Chabbert-Buffeta N, Skinner DC, Caraty A, Bouchard P. Neuroendocrine effects of progesterone. Steroids. 2000;65(10-11):613-620.

16. Richter TA, Robinson JE, Evans NP. Progesterone blocks the estradiol-stimulated luteinizing hormone surge by disrupting activation in response to a stimulatory estradiol signal in the ewe. Biol Reprod. 2002;67(1):119-125.

(c) 2018 The Author (s); This is an open-access article distributed under the terms of the Creative Commons Attribution License (http://creativecommons.org/licenses/by/4.0), which permits unrestricted use, distribution, and reproduction in any medium, provided the original work is properly cited. 